\title{
Surgical Treatment of Tarsal Tunnel Syndrome
}

\author{
Murat Gulcek ${ }^{1}$, Nural Aydin ${ }^{2}$, Ali Oznur ${ }^{3}$
}

\begin{abstract}
Background and aim: To assess the long-term functional outcomes of operative treatment for tarsal tunnel syndrome (TTS) and determine the factors that are associated with favorable and unfavorable clinical results.

Patients and methods: Sixty six patients (71 feet) who had undergone tarsal tunnel release (TTR) operation between 2000 and 2009 were recalled for a follow-up evaluation by a physiatrist to determine the outcome. The subjective complaints of the patients were re-evaluated using two different questionnaires: the foot function index (FFI) and a structured questionnaire.

Results: The mean age of the patients was $51.67 \pm 13.07$ (range 21-84) years. The mean follow-up time was 54.36 \pm 15.72 (range 12-96) months. Outcomes in terms of patient satisfaction were $45.1 \%$ — very satisfied (VS), 16.9\%—-moderately satisfied (MS), 19.7\%—somewhat satisfied (SS), and $18.3 \%$-not satisfied. Prognostic indicators of poor results in univariate analysis were older age, obesity, longer disease duration, coexisting lower back pain (LBP), plantar fasciitis, diabetes mellitus (DM), and/or carpal tunnel syndrome (all $p 0.05$ ).

Conclusion: LBP and DM were the most significant factors associated with unfavorable clinical results. The majority of the patients' symptoms improved following TTR operation.
\end{abstract}

Keywords: Patient outcome assessment, Surgery, Tarsal tunnel syndrome, Tibial nerve.

Journal of Foot and Ankle Surgery (Asia-Pacific) (2019): 10.5005/jp-journals-10040-1096

\section{INTRODUCTION}

Tarsal tunnel syndrome (TTS), which is currently a well-recognized source of foot dysfunction, is an entrapment neuropathy of the posterior tibial nerve (PTN) and its branches. TTS may produce physical discomfort both while walking and sleeping, and thereby impacts the patient's quality of life.

According to the literature, treatment outcomes are unsatisfactory and vary greatly. Several studies have investigated the outcomes and factors influencing the outcomes of TTR. ${ }^{1-7}$ Reports on the outcomes of TTS operation have varied based on the method of evaluation (specifically with regard to the definition of failure), small sample size, and the technique being evaluated. Sociodemographic characteristics (including sex, age, weight, as well as clinical and surgical factors) have been reported to influence outcomes in some studies, but not in others.

An accurate understanding of the factors predictive of the outcomes of the TTR would enable physicians and patients to make more informed decisions about whether to proceed with surgery and to develop more accurate expectations of the outcomes. The purpose of this study was to evaluate retrospectively the prognostic factors affecting the final outcome of surgical treatment of TTS. Additionally, we aimed to determine the factors that are associated with favorable and unfavorable clinical results.

\section{Patients and Methods}

Ethical permission and written informed patient consent were obtained for this study. A retrospective chart review was performed identifying 98 patients (105 feet) who had undergone a TTR procedure in our orthopedic department between 2000 and 2009. Of the 98 patients, 32 were lost to follow-up. Thus, the study group consisted of 66 patients (71 feet), providing a follow-up rate of $67 \%$. All procedures were performed by one subspecialty-trained orthopedic surgeon. The surgical technique was similar for all cases.
${ }^{1}$ Department of Orthopedics and Traumatology Clinic, Ankara Numune Education and Research Hospital, Ankara, Turkey

${ }^{2}$ Department of Physical Therapy and Rehabilitation, Faculty of Medicine, Guven Hospital, Ankara, Turkey

${ }^{3}$ Department of Orthopedics and Traumatology, Faculty of Medicine, Guven Hospital, Ankara, Turkey

Corresponding Author: Nural Aydin, Department of Physical Therapy and Rehabilitation, Faculty of Medicine, Guven Hospital, Ankara, Turkey, Phone: +90 532365 0474, e-mail: nalbayrak@yahoo.com

How to cite this article: Gulcek M, Aydin N, et al. Surgical Treatment of Tarsal Tunnel Syndrome. J Foot Ankle Surg (Asia-Pacific) 2019;6(1): 13-17.

Source of support: Nil

Conflict of interest: None

The decision of surgery was established based on the presence of all four indicators: (1) pain in the heel, forefoot, and midfoot; (2) a positive Tinels's sign at the tarsal tunnel; (3) an abnormal nerve conduction study of the posterior tibial, medial plantar, and lateral plantar nerves; (4) persistent symptoms despite 6 months conservative treatment. Exclusion criteria were clinical or electrophysiological evidence of accompanying conditions that could mimic TTS or interfere with its evaluation such as herniated spinal disc with neurological deficit, polyneuropathy with or without DM, stress fractures of the hindfoot, and inability to fill in the self-administered outcome questionnaire.

Imaging studies such as ultrasonography or magnetic resonance imaging were performed for identifying the spaceoccupying lesions, if any, around the PTN.

The subjective complaints of the patients were evaluated using two different questionnaires: the FFI index and a structured questionnaire. Both questionnaires were made at two different times. The first measurement was performed the day before the

() The Author(s). 2019 Open Access This article is distributed under the terms of the Creative Commons Attribution 4.0 International License (https://creativecommons. org/licenses/by-nc/4.0/), which permits unrestricted use, distribution, and non-commercial reproduction in any medium, provided you give appropriate credit to the original author(s) and the source, provide a link to the Creative Commons license, and indicate if changes were made. The Creative Commons Public Domain Dedication waiver (http://creativecommons.org/publicdomain/zero/1.0/) applies to the data made available in this article, unless otherwise stated. 
operation and the patient's baseline data were obtained. The second measurement was made via phone calls for all patients.

Patients were interviewed the day before surgery (baseline). At baseline, a structured questionnaire was used to record information on (1) demographic characteristics (patient age at the time of surgery and sex); (2) length of preoperative symptoms (months since surgery); (3) body mass index (BMI); (4) side of extremity involvement; (5) chronic LBP unrelated to TTS; (6) preexisting co-morbidities at baseline (DM, carpal tunnel syndrome (CTS), trauma, pes planus (PP), plantar fasciitis (PF), and Morton's neuroma (MN)); (8) subjective symptom characteristics (type: burning, cramping, tingling, numbness, paresthesias, night pain, continuous pain, daytime pain, and time of the predominant sensory symptoms).

The patients were questioned on eight possible subjective symptoms. These are burning, cramping, tingling, numbness, paresthesias, night pain, continuous pain, and daytime pain. If so, the time was saved. These questions were also asked via phone during the last checks.

A FFI was developed to measure the impact of foot pathology on functions in terms of pain, disability, and activity restriction. The FFI is a self-administered index consisting of 23 items divided into 3 subscales. ${ }^{3,8}$

The patients were asked to choose an answer for each question based on their worst preoperative condition. The answer was to reflect their symptoms for a typical 24-hour day over a 2-week period. An independent researcher was present to provide aid, if necessary, for patients answering the questionnaire. Follow-up interviews were made by an objective physiatrist. Patients were contacted via telephone. At a follow-up interview, FFI was reevaluated. Response to clinical change was assessed by comparison of the pre-operative and post-operative scores.

All patients were asked to grade their overall satisfaction with surgical result as VS-indicating complete symptom resolution, no functional limitations, no analgesic medications being taken; MS -indicating marked improvement with minor symptoms that do not interfere with function, pain medication not being taken regularly; SS-indicating some decrease in symptom intensity, pain and functional impairment occurring regularly, and pain medications being taken regularly; and not satisfied (NS)-indicating no improvement, difficulty with all activity or worsening of symptoms. ${ }^{3}$ In addition, the length of follow-up, the type of complications (if any) after discharge (infection, deep venous thrombosis, and others [yes/ no]), and information regarding whether the patient underwent a revision surgical procedure were evaluated.

\section{Surgical Technique}

The surgical technique for TTR was performed under general endotracheal or regional anesthesia by a single surgeon and was similar for all patients. The tarsal tunnel on the affected extremity was approached through a curved incision that begins $10 \mathrm{~cm}$ proximal to the posterior aspect of the medial malleolus and was extended down to $2 \mathrm{~cm}$ posterior to the posterior margin of the tibia. In order to identify flexor retinaculum, the incision was deepened through the subcutaneous tissue and fat. The retinaculum was released in a proximal to distal direction. Just because the retinaculum distally might be extremely taut and dense, a curved clamp was placed between the retinaculum and underlying tissues so as not to injure other vital structures. PTN beneath the flexor retinaculum was identified and traced distally through the tarsal tunnel, where branching into three terminal branches occurs. Medial and lateral plantar nerves (including the first branch of the lateral plantar nerve), as well as the medial calcaneal nerve were released into their respective tunnels, and the septum between the tunnels was resected. Once PTN and its terminal branches were released, the pneumatic tourniquet, if one was used, was deflated. We observed the nerve carefully to determine whether capillary filling was adequate along the course of the nerve. We did not perform neurolysis typically not to make the nerve more vulnerable to scarring. After bleeding control, we closed the subcutaneous tissue and skin in layers, without closing the retinaculum.

\section{Statistical Analysis}

A total of 71 feet achieved $80.3 \%$ power to detect a significant difference of -16.9 between the null hypothesis that the group FFI mean is $17.3 \pm 22.4$ (for patients who have LBP) and the alternative hypothesis that the FFI mean is $34.2 \pm 18.7$ (for patients who don't have LBP) with a significance level (alpha) of 0.05 using a twosided two-sample $t$ test. The Kolmogorov-Smirnov goodness-offit test is performed to check whether the data follow a normal distribution. Associations between the principal outcomes at follow-up and preoperative predictor variables were examined with the independent $t$ test for categorical variables and the Pearson correlation for continuous variables. The paired $t$ test is used for comparing preoperative and follow-up outcomes. In order to examine the effect of the BMI and symptom characteristics on principal outcomes (FFI scores), we performed a one-way analysis of variance with Tukey's HSD multiple comparison test.

A multiple linear regression model is fitted following the univariate analysis of principal outcomes. We introduced all predictor variables with univariate significance levels less than $0.10(p<0.10)$ into multiple linear regression models. We initially modeled individual variables (DM, trauma, PP, PF, MN, LBP, and symptom characteristics) against each outcome. We then took all of the significant predictors of outcome and advanced them to the final models. We ran final models with and without stepwise selection procedures. These two approaches identified almost the same statistically significant predictor variables, with a generally similar magnitude of effect.

Univariate data searching relevance between patient satisfaction and predictor variables were analyzed by the Chisquare test. Then, the multinomial logistic regression model is fitted for patient satisfaction following the univariate analyses. In the multinomial logistic regression, the outcome category NS for patient satisfaction is taken as the reference category. Odds ratios, standard errors, and model goodness-of-fit measures are reported.

\section{Results \\ Demographic Outcomes}

The mean duration of symptoms of $30.85 \pm 32.49$ (range 6-150) months. The mean duration of follow-up was $54.36 \pm 15.72$ (range 12-96) months (Table 1).

Table 1: Patient characteristics

\begin{tabular}{llll}
\hline & Female & Male & Total \\
\hline$N$ & $54(81.8 \%)$ & $12(18.2 \%)$ & 66 \\
$\begin{array}{l}\text { Age } \\
\text { (mean-range) }\end{array}$ & $51.92(21-84)$ & $50.5(34-75)$ & $51.67(21-84)$ \\
$\begin{array}{l}\text { Side (right/ } \\
\text { left/bilateral) }\end{array}$ & $38 / 11 / 5$ & $8 / 4 / 0$ & $46 / 15 / 5$ \\
$\begin{array}{l}\text { BMl } \\
\text { (mean-range) }\end{array}$ & $27.82(18-44.4)$ & $27.72(25.4-31.3)$ & $27.8(18-44.4)$ \\
\hline
\end{tabular}




\section{Pre and Postoperative Symptom Outcomes}

Pre and postoperative symptom characteristics and descriptive statistics for FFI are described in Tables 2 and 3, respectively. Outcomes in terms of patient satisfaction were $45.1 \%$ (32/71) VS, $16.9 \%(12 / 71)$ MS, $19.7 \%(14 / 71)$ SS and $18.3 \%$ (13/71) NS results.

\section{Univariate Analysis of Outcomes}

Outcomes did not vary by sex and/or side of extremity involvement (all $p>0.05$ ). The duration of preoperative symptoms had a significant correlation with preoperative and postoperative total FFI scores (correlation coefficient $r=0.37, p=0.001$ and $r=0.48$, $p<0.001$, respectively). Age had a significant correlation with postoperative total FFI scores (correlation coefficient $r=0.30$, $p=0.01$ ), but no correlation with preoperative total FFI scores (correlation coefficient $r=0.12, p=0.31$ ). There was no significant correlation between the duration of follow-up and preoperativepostoperative total FFI scores (correlation coefficient $r=0.04, p=$ 0.69 and $r=0.09, p=0.42$, respectively).

All univariate analyses between preexisting co-morbidities and outcomes are given in Table 4. Symptom characteristics did not create any significant differences in pre and postoperative FFI scores (all $p>0.05$ ). BMI groups (normal-overweight and obese) have significantly different postoperative disability scores $(p=0.040)$, and obese patients had higher postoperative disability scores than normal-weight patients ( $p=0.032$ ).

Patients with high surgery satisfaction were less likely to have co-morbid DM, PF, CTS and LBP (Chi-square: $p<0.001, p=0.005$, $p=0.013$ and $p<0.001$, respectively).

\section{Multivariate Analysis of Outcomes}

On the multiple linear regression, age, BMI, symptom characteristics, preoperative symptom duration, PF, and CTS were not significant prognostic factors (all $p>0.05$ ) and were excluded. The other remaining two factors (LBP, DM) were still significant prognostic factors for worse outcomes following surgery. The regression coefficient for LBP was -14.63 with a standard error of 4.89

Table 2: Pre and postoperative symptom characteristics

\begin{tabular}{lcc}
\hline & Preoperative (/71) & Postoperative (/71) \\
\hline Burning & 22 & 17 \\
Cramping & 23 & 10 \\
Tingling & 6 & 1 \\
Numbness & 20 & 15 \\
Paresthesias & 64 & 33 \\
Night pain & 11 & 5 \\
Continuous pain & 33 & 16 \\
Daytime pain & 3 & 1 \\
\hline
\end{tabular}

Table 3: Descriptive statistics for the FFI

\begin{tabular}{llll}
\hline & $\begin{array}{l}\text { Pre-op } \\
(\text { mean } \pm \text { SD) }\end{array}$ & $\begin{array}{l}\text { Follow-up } \\
(\text { mean } \pm \text { SD })\end{array}$ & p value \\
\hline Mean pain subscore & $59.60 \pm 19.45$ & $32.40 \pm 28.70$ & $p<0.001$ \\
$\begin{array}{l}\text { Mean activity } \\
\text { limitations subscore }\end{array}$ & $36.71 \pm 22.03$ & $14.87 \pm 18.24$ & $p<0.001$ \\
$\begin{array}{l}\text { Mean disability } \\
\text { subscore }\end{array}$ & $53.29 \pm 21.75$ & $27.61 \pm 25.23$ & $p<0.001$ \\
Mean total FFI score & $49.87 \pm 18.05$ & $24.96 \pm 22.33$ & $p<0.001$ \\
\hline
\end{tabular}

$(p=0.004)$ and for DM was -14.19 with a standard error of 5.96 $(p=0.020)$. The linear regression model was significant $(p<0.001)$ and the multiple correlation coefficient was $R=0.457$.

Gender, BMI, side of extremity involvement, symptom characteristics, co-morbid MN, PP, and paresthesia have no significant effects on patient satisfaction. However, preoperative symptom duration, DM, and LBP had significant effects on patient satisfaction (Table 5). For example, a decrease in preoperative symptom duration had significantly improved the satisfaction (from NS to VS category) by 1.04 times, having DM and LBP had decreased the patient satisfaction by 0.021 and 0.019 times (multinomial logistic regression, $\chi^{2}=59.89, p<0.001$, Nagelkerke $R^{2}=0.616$ ).

There were no surgery-related complications. Only one patient required a revision TTR for recurrent symptoms.

\section{Discussion}

There is no consensus in the literature as to what parameters will predict the outcome of TTS surgical treatment. From this respect, we aimed to assess the long-term patient-relevant outcomes of TTR operation and to identify factors influencing outcomes. We found that FFI significantly improved after 4.5 years. Furthermore, patients with DM and LBP that had undergone TTR operation had a significantly worse long-term outcome than those without DM and LBP.

Gondring et al. reported that positive results are obtained from both objective and subjective outcome analyses of surgical treatment of TTS. However, the patient's expectations were higher than the surgeon's. ${ }^{2}$ For this reason, we evaluated the clinical results of the patients with the FFI questionnaire.

There is a slightly higher incidence of TTS in females compared to in males. ${ }^{9}$ In our study, there were 54 women and 12 men.

Takakura concluded that the outcomes of TTR are generally excellent in younger patients, but outcomes are generally less favorable in patients with prolonged symptoms. ${ }^{10}$ Since chronic pain has a direct impact on most of the daily activities, it is an important aspect in the outcome of TTS surgery. Franson reported that chronic symptoms of TTS had a lower rate of positive surgical outcomes. He recommended that in patients who do not respond to conservative treatment in the short term, surgical intervention should be considered. ${ }^{11}$ In another study, Reichert described the importance of immediate diagnosis and short period between occurrences of symptoms in surgical treatment for better outcomes. $^{12}$

In our study, clinical success after surgical treatment was found to be low in patients with a long symptom duration. DM and LBP are chronic diseases that can affect the neural tissue in patients where the clinical outcome is a failure. ${ }^{13,14}$ Symptoms are long term in these chronic diseases where pathology effects on the nerve tissue are known, and the clinical success after surgical treatment may be low owing to possible neuropathologies.

Moreover, Gondring et al. indicated that the increased incidence of LBP might be observed in unimproved patients. ${ }^{2}$ Although coexisting LBP and DM are the strongest predictors of a less-favorable outcome of surgery in our study, it should be noted that PTN decompression surgery in TTS patients with diabetic neuropathy reduces pain, improves plantar sensitivity, and prevents foot ulcers and lower leg amputations. Therefore, TTR should be considered in patients with diabetic peripheral neuropathy for whom conservative and/or medical treatment failed, in order to improve the patients' quality of life..$^{17,18}$ 


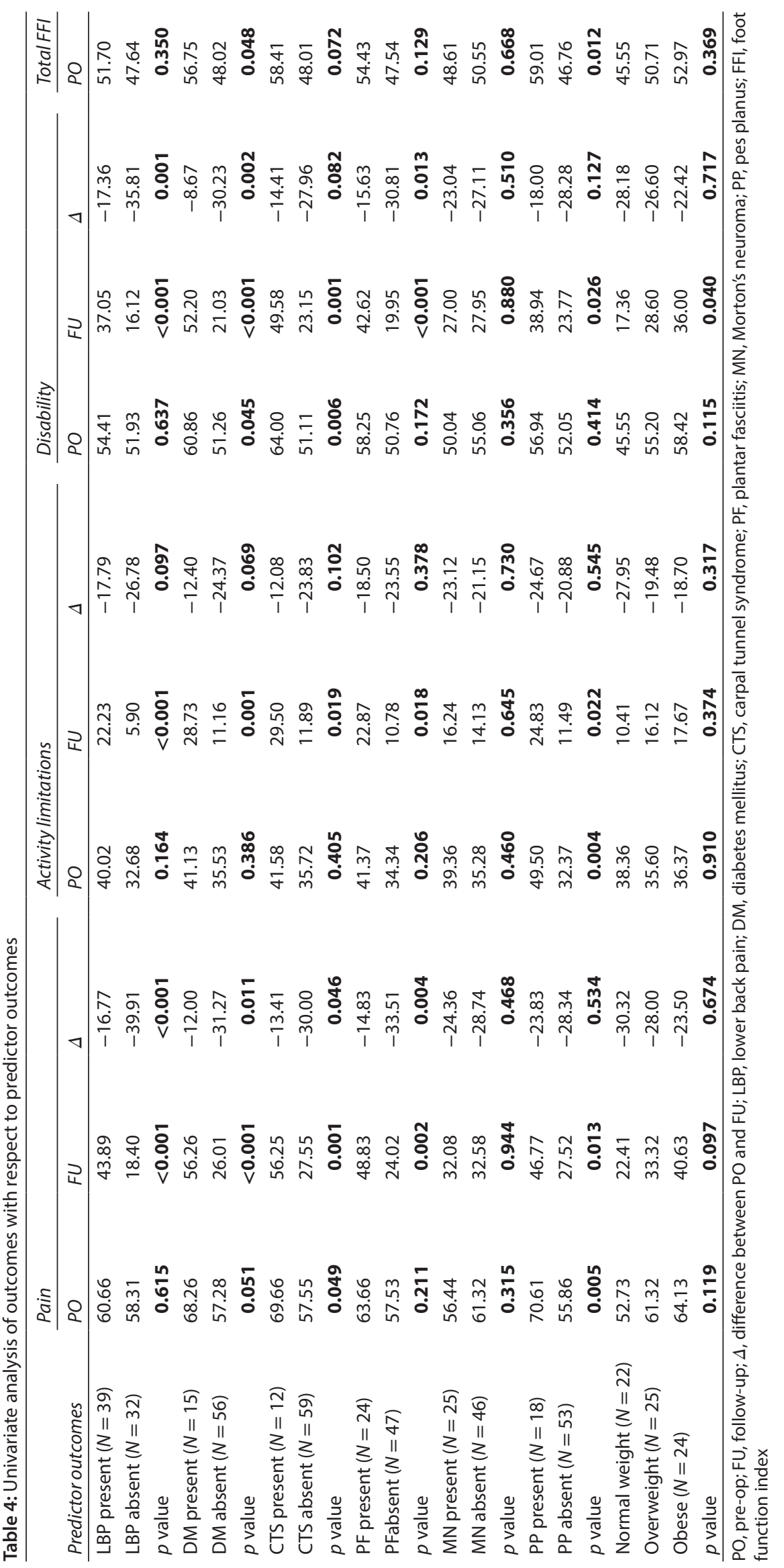


Table 5: Multinomial logistic regression results

\begin{tabular}{llrcl}
\hline $\begin{array}{l}\text { Outcome } \\
\text { category }\end{array}$ & Variables & Beta (std.error) & OR & p value \\
\hline VS & Intercept & $8.496(3.391)$ & NA & 0.012 \\
& Age (years) & $-0.045(0.047)$ & 0.956 & 0.339 \\
& Preop süre & $-0.036(0.018)$ & 0.965 & $\mathbf{0 . 0 4 3}$ \\
& DM & $-3.882(1.658)$ & 0.021 & $\mathbf{0 . 0 1 9}$ \\
& CTS & $-0.502(1.718)$ & 0.606 & 0.770 \\
& PF & $0.080(1.244)$ & 1.083 & 0.949 \\
MS & LBP & $-3.973(1.737)$ & 0.019 & $\mathbf{0 . 0 2 2}$ \\
& Intercept & $4.720(3.426)$ & NA & 0.168 \\
& Age (years) & $0.004(0.048)$ & 1.004 & 0.933 \\
& Preop süre & $-0.040(0.022)$ & 0.961 & 0.063 \\
& DM & $-3.474(1.672)$ & 0.031 & $\mathbf{0 . 0 3 8}$ \\
& CTS & $1.042(1.558)$ & 2.835 & 0.504 \\
& PF & $-0.326(1.328)$ & 0.722 & 0.806 \\
SS & LBP & $-3.164(1.756)$ & 0.042 & 0.072 \\
& Intercept & $1.730(3.353)$ & NA & 0.606 \\
Age (years) & $0.018(0.044)$ & 1.018 & 0.682 \\
& Preop süre & $-0.012(0.015)$ & 0.988 & 0.437 \\
DM & $-4.680(1.793)$ & 0.009 & $\mathbf{0 . 0 0 9}$ \\
& CTS & $2.114(1.548)$ & 8.283 & 0.172 \\
PF & $1.342(1.206)$ & 3.827 & 0.266 \\
LBP & $-1.972(1.835)$ & 0.139 & 0.283 \\
\hline
\end{tabular}

The reference category is NS, not satisfied; VS, very satisfied; MS, mostly satisfied; SS, satisfied. TTS, Tarsal tunnel syndrome; PTN, posterior tibial nerve; TTR, tarsal tunnel release; BMI, body mass index; LBP, lower back pain; DM, diabetes mellitus; CTS, carpal tunnel syndrome; trauma; PP, pes planus; PF, plantar fasciitis; MN, Morton's neuroma

We also found a significant correlation between age and postoperative total FFI score. Furthermore, in this study, there were significant correlations between the duration of preoperative symptoms and pre and postoperative total FFI scores. This is contrary to what Gondring reported, in which the time length of the patient's complaints was determined not to be an applicable indicator because of the difficulty in accurately measuring the actual onset of the syndrome. ${ }^{2}$

One of the key results of our work is the statistics that symptom characteristics had virtually no association with surgical outcomes.

Sammarco reviewed 72 patients (108 feet) with this condition for 12 years, with a mean follow-up of 58 months evaluating their functional outcome, and discovered that the majority of the patients' so-called idiopathic condition substantially arose from a vascular origin. Chronic edema from prolonged standing without the need to be varicose veins and microedema of the PTN create a combined effect over a period of several years. ${ }^{4}$ This may be an explanation as to why patients with TTS of unknown etiology do poorly after surgical decompression.

Budak et al. reported the presence of compression neuropathy of the medial and lateral plantar nerve in PP subjects. ${ }^{15}$ Tibial nerve tension is increased in an unstable foot during eversion, dorsiflexion, combined dorsiflexion-eversion, and cyclical load with increasing internal rotation. ${ }^{16}$ In our study, the reported long-term postoperative intensity of the pain was lower in non-PP patients like Budak's study.

There was no significant association of TTS with CTS in multivariate analysis, as other authors have speculated. ${ }^{17}$ Bailie et al. reported this rate as $72 \% .^{3}$
Patients were satisfied with the surgical outcome in $62 \%$ of the cases. Postoperative improvement in the median FFI score reflected this satisfaction as well. ${ }^{3}$

The study has several limitations. Firstly, due to the longterm follow-up, the drop out rate was high. Another limitation is the method of measuring the quality of life. A TTS-specific questionnaire would be preferable. The lack of control group makes it difficult to state that surgical decompression solely caused this clinical improvement.

\section{Conclusion}

Long-term follow-up outcome results of our study show that TTR is a safe procedure with a high satisfaction rate. However, it should be kept in mind that the success rate in patients with DM and LBP is lower than those without DM and LBP.

\section{References}

1. Turan I, Rivero-Melián C, et al. Tarsal tunnel syndrome. Outcome of surgery in longstanding cases. Clin Orthop Relat Res 1997;343: 151-156. DOI: 10.1097/00003086-199710000-00025.

2. Gondring WH, Shields $B$, et al. An outcomes analysis of surgical treatment of tarsal tunnel syndrome. Foot Ankle Int 2003;24(7):545-550.

3. Bailie DS, Kelikian AS. Tarsal tunnel syndrome: diagnosis,surgical technique and functional outcome. Foot Ankle Int 1998;19(2):65-72. DOI: $10.1177 / 107110079801900203$.

4. Sammarco GJ, Chang L. Outcome of surgical treatment of tarsal tunnel syndrome. Foot Ankle Int 2003;24(2):125-131. DOI: $10.1177 / 107110070302400205$.

5. Urgüden M, Bilbaşar $\mathrm{H}$, et al. Tarsal tunnel syndrome- the effect of the associated features on outcome of surgery. Int Orthop 2002;26(4):253-256. DOI: 10.1007/s00264-002-0351-7.

6. Herbsthofer B, Vogt T, et al. Diagnosis and long-term outcome after surgical therapy of tarsal tunnel syndrome. $Z$ Orthop Ihre Grenzgeb 1998;136(1):77-82. DOI: 10.1055/s-2008-1051287.

7. Mondelli M, Giannini F, et al. Clinical and electrophysiological findings and follow-up in tarsal tunnel syndrome. Electroencephalogr Clin Neurophysiol 1998;109(5):418-425. DOI: 10.1016/S0924980X(98)00039-3.

8. Skalley TC, Schon LC, et al. Clinical results following revision tibial nerve release. Foot Ankle Int 1994;15(7):360-367. DOI: 10.1177/107110079401500703.

9. Lau JT, Daniels TR. Tarsal tunnel syndrome: a review of the literature. Foot Ankle Int 1999;20(3):201-209. DOI: 10.1177/107110079902000312.

10. Takakura Y, Kitada C, et al. Tarsal tunnel syndrome. Causes and results of operative treatment. J Bone Joint Surg Br 1991;73:125-128. DOI: 10.1302/0301-620X.73B1.1991745.

11. Franson J, Baravarian B. Tarsal tunnel syndrome: a compression neuropathy involving four distinct tunnels. Clin Podiatr Med Surg 2006;23:597-609. DOI: 10.1016/j.cpm.2006.04.005.

12. Reichert $\mathrm{P}$, Zimmer $\mathrm{K}$, et al. Results of surgical treatment of tarsal tunnel syndrome. Foot Ankle Surg 2015;21(1):26-29. DOI: 10.1016/ j.fas.2014.08.013.

13. Mazilu G, Budurca RA, et al. Surgical treatment of tarsal tunnel syndrome in diabetic neuropathy. Rev Med Chir Soc Med Nat lasi 2012;116(1):128-134.

14. Gondring WH, Tarun PK, et al. Touch pressure and sensory density after tarsal tunnel release in diabetic neuropathy. Foot Ankle Surg 2012;18(4):241-246. DOI: 10.1016/j.fas.2012.02.001.

15. Budak F, Bamaç B, et al. Nerve conduction studies of lower extremities in pes planus subjects. Electromyogr Clin Neurophysiol 2001;41(7):443-446.

16. Daniels TR, Lau JT, et al. The effects of foot position and load on tibial nerve tension. Foot Ankle Int 1998;19(2):73-78. DOI: 10.1177/107110079801900204

17. Paterson DC. Bilateral carpal and tarsal tunnel syndrome. Med J Aust 1966 Aug 27;2(9):421. 